\title{
XXII.
}

Aus dem pharmakologischen Institut zu Marburg.

\section{Veber das Protopin.}

\author{
Von \\ Dr. R. v. Engel.
}

(Mit 9 Curven.)

Das Alkaloid Protopin - von der Zusammensetzung $\mathrm{C}_{20} \mathrm{H}_{19} \mathrm{NO}_{5}$ - wurde 1870 von Hesse im Opium nachgewiesen. Es ist in demselben in äusserst geringer Menge vorhanden: $\mathrm{Hesse}^{1}$ ) erhielt ca. $1,5 \mathrm{~g}$ bei einer Ausbeute ron $80 \mathrm{~g}$. Cryptopin, Merck $50 \mathrm{~g}$ Protopin gegen $11 / 2$ Kilo Cryptopin und $150 \mathrm{~g}$ Laudanosin.

Hingegen haben neuere Untersuchungen eine weitere Verbreitung des Protopins erwiesen: Eykman ${ }^{2}$ ) fand es in der Macleya cordata, Selle ${ }^{3}$ ) im Chelidonium majus, ferner in Stylophoron diphyllum einen dem Protopin anscheinend sehr nahe stehenden, vielleicht damit identischen Körper; während über das Vorkommen des Protopins in Sanguinaria canadensis gegenwärtig Untersuchungen angestellt werden. : Sämmtliche genannten Pflanzen gehören der Familie der Papaveraceen an, so dass Selle zu dem Schluss gelangt, „dass dieses schon in einigen Papaveraceen gefundene Alkaloid vielleicht von allgemeiner Bedeutnng für diese Pflanzenfamilie ist". Gerade dieser Umstand liess eine physiologische Untersuchung des Alkaloides nicht unerwünscht erschèinen.

Verwendet wurde zu den folgenden Versuchen Protopin aus Chelidonium und aus Opium. Das erstere stammte aus dem pharmaceutischchemisschen Institut des Herrn Prof. S chmid t (Sell e's Darstellung), bedurfte also keiner weiteren Prufung; das letztere wurde von Herrn

1) Annalen für Chemie und Pharmacie. Supplementband VIII.

2) Pharm. Jap. trans.; citirt nach Fehling, Handwörterbuch der Chemie. V. Bd. S. 10.

$\because$ 3) Ueber die Alkaloide der Wurzeln von Stylophoron diphyllum und Chelidonium majus; Inaug.-Diss. Erlangen 1889. 
Merck freundlichst zur Verfügung gestellt. Die Prifung der chemischen Reinheit dieser Substanz ergab ein befriedigendes Resultat. Der Schmelzpunkt wurde bei 202 - corrigirt - gefunden; nach Hesse liegt er bei 202, nach Eykman bei 201; auch Selle fand ihn für das Chelidoniumprotopin bei $205^{\circ}$ uncorrigirt. Mit Schwefelsäure erhielt man die charakteristische Protopinreaction: anfängliche Gelb-, darauf folgende Schwarzfärbung der Protopinkrystalle, während die Schwefelsäure von violetten Streifen durchzogen war. Es wurden endlich zwei Doppelsalze dargestellt: salzsaures Protopingoldchlorid und salzsaures Protopinplatinchlorid. Das letztere zeigte völlig das von Hesse angegebene Verhalten: man erbält es aus der wasserklaren salzsauren Lösung als gelben krystallinischen Niederschlag, der sich sehr gut mit Wasser auswaschen lässt und dann beim Trocknen ein dunkelgelbes Pulver darstellt. Dasselbe löste sich in überschiussigem Alkohol erst bei intensivem Erhitzen. Nach tagelangem Stehen schied sich eine kleine Quantität glänzender Krystallnadeln ans, die zu einer Analyse nicht geniigte. Der Rest erschien in Form weisser Schollen.

Das salzsaure Protopingoldchlorid wurde als dunkelorangefarbener krystallinischer Niederschlag erhalten; derselbe ist in kaltem Wasser unlöslich, in kochendem Wasser zersetzt er sich unter Reduction des Goldes. Der Niederschlag wurde nun mit Wasser gewasehen, in kochendem Alkohol von 96 Proc. gelöst, filtrirt. Nach 2 tägigem Stehen schied sich das salzsaure Protopingoldchlorid als schön rubinrothe, krystallinische Masse am Boden und an der Wand des Glases aus. Zur Ausfuhrung der Gewichtsanalyse wurde das Gold durch Magnesiumpulver reducirt, das Chlor durch Titrirung bestimmt und durch nachträgliche Wägung controlirt. Es ergab:

$$
\begin{aligned}
& \text { 0,3025 Substanz . . . . 0,0910 Au }=28,39 \text { Proc. } \\
& 0,3025 \text { Substanz . . . } 0,0655 \mathrm{Cl}=20,43 \text { Proc. }
\end{aligned}
$$

Selle (a. a. 0.) erbielt für die entsprechende Verbindung des Stylophorins $\mathrm{C}_{20} \mathrm{H}_{19} \mathrm{NO}_{5}+\mathrm{H}_{2} \mathrm{O} ; \mathrm{Au}=28,39$ Proc.; $\mathrm{Cl}=20,49$ Proc.

Es konnte daher die chemische Reinheit der Substanz als erwiesen betrachtet werden; auch ergab die Untersuchung, dass beide Protopine in ihren Wirkungen identisch sind.

Die Versuche zur Ermittlung der physiologischen Wirkung wurden an Fröschen beider Arten, an Meerschweinchen, Kaninchen und Katzen ausgefübrt.

\section{Versuche an Fröschen.}

Die beiden Arten von Rana - temporaria und esculenta - verhalten sich gegen das Protopin gleichmässig. Die Dosis letalis liegt 
bei $7-8 \mathrm{mg}$. Folgende Versuche mögen die Erörterung der Wirkung illustriren.

\section{Versueb I. 24. April.}

12 h. 14 m. Einer Essculenta, kräftigem Männchen, wurden 2 mg Protopin (aus Opium) in den Rückenlymphsack injicirt.

12 h. $40 \mathrm{~m}$. Der Frosch bleibt, frei auf den Tisch gesetzt, ruhig sitzen; die weggezogene Extremität zieht er erst nach einer Weile und unter zuckenden Bewegungen an.

12 h. 45 m. Erträgt die Rückenlage, aus der er sich erst nach starken Reizen unter lebhaften Anstrengungen umdreht. Nach starken mechanischen Reizen oder auf Benetzen mit Essigsäure macht er noch einen einzelnen ungeschickten Sprung.

2 h. $20 \mathrm{~m}$. Die Erscheinungen sind geschwunden. Frosch normal.

Versuch II. 17. April.

12 h. $37 \mathrm{~m}$. Einer Temporaria (männlich) werden $4 \mathrm{mg}$ Opiumprotopin in den Riùckenlymphsack injicirt.

12 b. $39 \mathrm{~m}$. Sitzt ruhig da; auf Reize macht der Frosch einen ungeschickten Sprung, wobei er seitlich umfällt, oder er versucht in unbehülfficher Weise fortzukriechen.

$12 \mathrm{~h} .42 \mathrm{~m}$. Fortbewegung unmöglich; auch auf sehr intensive Reize erfolgen blos stossende Bewegungen der hinteren Extremitäten; ebensowenig vermag sich der Frosch aus der Rückenlage zu befreien. Beflexerregbarkeit erhalten.

12 h. $45 \mathrm{~m}$. Die elektrische Erregbarkeit, sowohl vom Riickenmark aus, als bei directer Reizung der Oberschenkelmusculatur sehr stark herabgesetzt; dabei sind die Contractionen unregelmässig auf einzelne Muskelbündel beschränkt, eigenthümlich zitternd, wellenfờrmig.

\section{Versuch III. 25. April.}

$11 \mathrm{~h} .25 \mathrm{~m}$. Einem Temporaria-Männchen $6 \mathrm{mg}$ Opiumprotopin in den Rückenlymphsack.

11 h. $31 \mathrm{~m}$. Auf Reize nur mehr stossende Bewegungen in den hinteren Extremitäten.

11 h. $32 \mathrm{~m}$. Erträgt Riickenlage.

$11 \mathrm{~h} .36 \mathrm{~m}$. Liegt vollkommen regungslos auf dem Rücken. Die Athmung ist sistirt. Reflexerregbarkeit normal; bei wiederholten stärkeren Reizen treten 2-3 stossende Bewegungen ein, die jedoch nur von momentaner Dauer sind und nach deren Verschwinden durch längere Zeit überhaupt keine Reflexbewegung auszulösen ist. tion mehr.

$11 \mathrm{~h} .45 \mathrm{~m}$. Cornealreflex erloschen; auf Essigsäure keine Reacerhalten.

$11 \mathrm{~h} .47 \mathrm{~m}$. Auch anf mechanische Reize keine Reaction mehr zu

$2 \mathrm{~h} .50 \mathrm{~m}$. Vom Rückenmark aus erst bei 8 Rollen Distanz mit dem Inductionsstrome schwache Zuckung za erhalten. Complete Lähmung und Erloschensein aller Reflexe; das blossgelegte Herz pulsirt äusserst schwach. 
Aus dem Dargelegten geht hervor, dass es sich bei kleinen Dosen - von $2 \mathrm{mg}$ - um die bekannte narkotische Wirkung der meisten Opiumalkaloide handelt. Steigert man jedoch die Dose auf $4 \mathrm{mg}$ und böher, so drängen sich Erscheinungen in den Vordergrund, welche das Bild einer mehr oder minder stark ausgebildeten Lähmung hervorrufen und auf den ersten Blick den Eindruck einer curareartigen Wirkung machen. Um nun von vornherein zu entscheiden, ob man es mit einer centralen oder peripheren Wirkung za thun babe, warde eine Reihe von Versuchen angestellt, bei denen ein Bein aus der Circulation ausgeschlossen wurde.

\section{Versueh IV. 1. April.}

5 h. $58 \mathrm{~m}$. Einer Temporaria, männlich, wird die rechte Arteria iliaca unmittelbar unterhalb der Theilung unterbunden, das rechte Lymphherz am Steissbein zerstört.

$6 \mathrm{~h} .6 \mathrm{~m}$. Injection von $5 \mathrm{mg}$ Chelidoniumprotopin in die beiden Seitenlymphsäcke.

$6 \mathrm{~h} .30 \mathrm{~m}$. Die lähmende Wirkung ist eingetreten.

6 h. $32 \mathrm{~m}$. Prüfung der elektrischen Erregbarkeit bei directer Muskelreizung mit dem Inductionsstrom, 12. Rollen Distanz: Rechts straffe danernde Contraction, echter Tetanus, links intermittirende, rasch sich folgende, wellenförmige Contractionen.

6 h. $40 \mathrm{~m} .10$ Rollen Distanz: Rechts unveränderter Tetanus, links streckt sich das Bein einen Moment, um sofort wieder zu erschlaffen, während in den einzelnen Muskelbündeln springende, wurmförmige Contractionen auftreten. Vom Rückenmark aus rechts kräftiger Tetanus, links schleudernde Bewegung des Beines.

2. April. Frosch vollständig gelähmt, elektrische Prüfung ergiebt rechts schwachen, aber immer noch deutlichen Tetanus, links vibrirende Bewegungen einzelner Bündel.

Es handelt sich demnach um eine peripbere Wirkung des Giftes, und zwar ist dieselbe rornehmlich auf die Muskelsubstanz gerichtet; um diese Wirkung nun näher prüfen zu können, wurden mehrere Versuche am Myographion vorgenommen. Es wurde zu diesem Zwecke einem Frosche die eine Iliaca knapp unterhalb der Theilung doppelt unterbunden und durchtrennt, beide Plexus armirt und nach Eintritt der Vergiftungserscheinungen durchtrennt; hierauf beide Gastrocnemii mit langer Sehne freipräparirt und in directe Verbindung mit der Leitung gesetzt. Zwei Versuche ergaben folgende Curven $1-4$ (S.423).

$$
\text { Versuch V. }
$$

Temporaria, linke lliaca mit dem linken Plexus doppelt unterbunden und durchtrennt, rechter Plexus armirt.

$3 \mathrm{~h} .52 \mathrm{~m}$. Injection von $7 \mathrm{mg}$ Protopin (Chelidonium) in den Bauchlymphsack. 
4 h. 8 m. Eintritt der Vergiftungserscheinungen.

4 h. $16 \mathrm{~m}$. Rechter Plexus durchtrennt. Präparirung beider Gastrocnemii, Anbringung am Myographion.

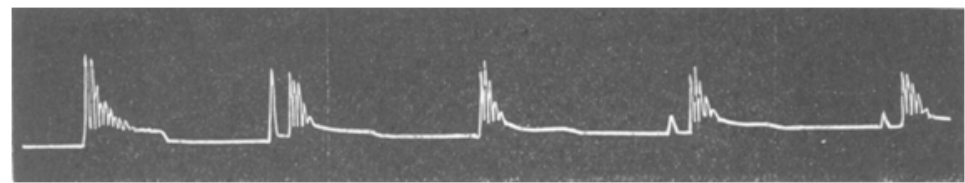

Curve 1. Veryifteter Gastrocnemius bei 10 Rollen Abstand. 4 b. 20) m.

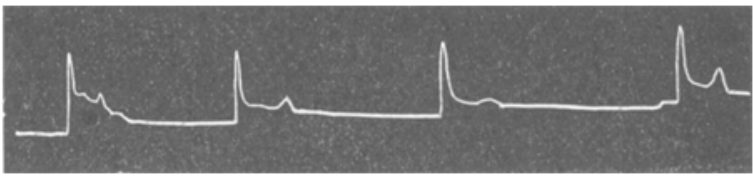

Curve 2. 5 h. Vergifteter Gastrocnemius bei 5 Rollen Abstand.

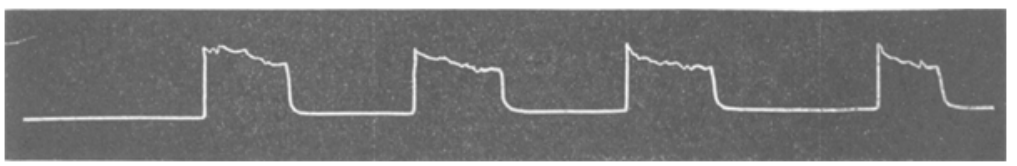

Curve 3. Linker (unvergifteter) Gastrocnemius, bei 11 Rollen Abstand. $4 \mathrm{~h} .25 \mathrm{~m}$.

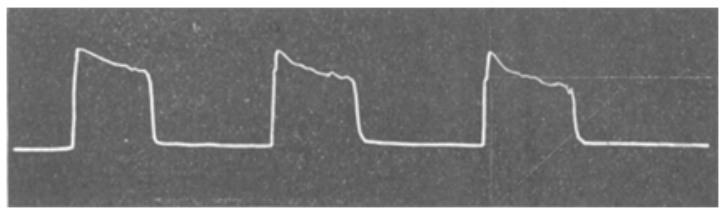

Curve 4. 5 h. 2 m. Linker (unvergifteter) Gastrocnemius bei 8 Rollen Abstand.

\section{Versuch VI.}

Esculenta, linke Iliaca unterbunden,-Plexus beiderseits armirt.

$11 \mathrm{~h} .2 \mathrm{~m} .6 \mathrm{mg}$. Protopin (Opium) injicirt in beide Lymphsäke. Nach Eintritt der Vergiftungserscheinungen beide Plexus durchtrennt.

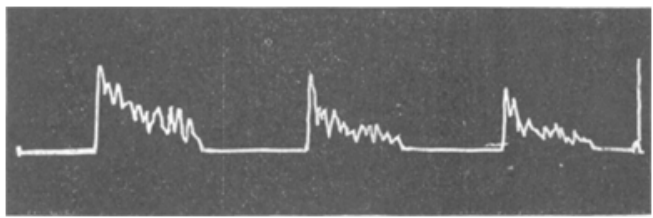

Cbrve 5. Vergifteter Muskel bei 12 Rollen. $50 \mathrm{~g}$ Belastung.

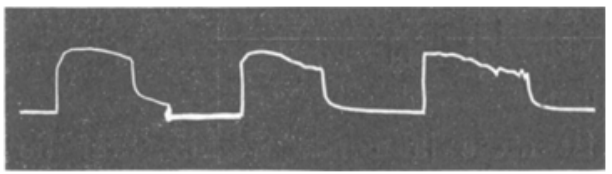

Curre 6: Gesunder Muskel bei 12 Rollen. $50 \mathrm{~g}$ Belastung. 

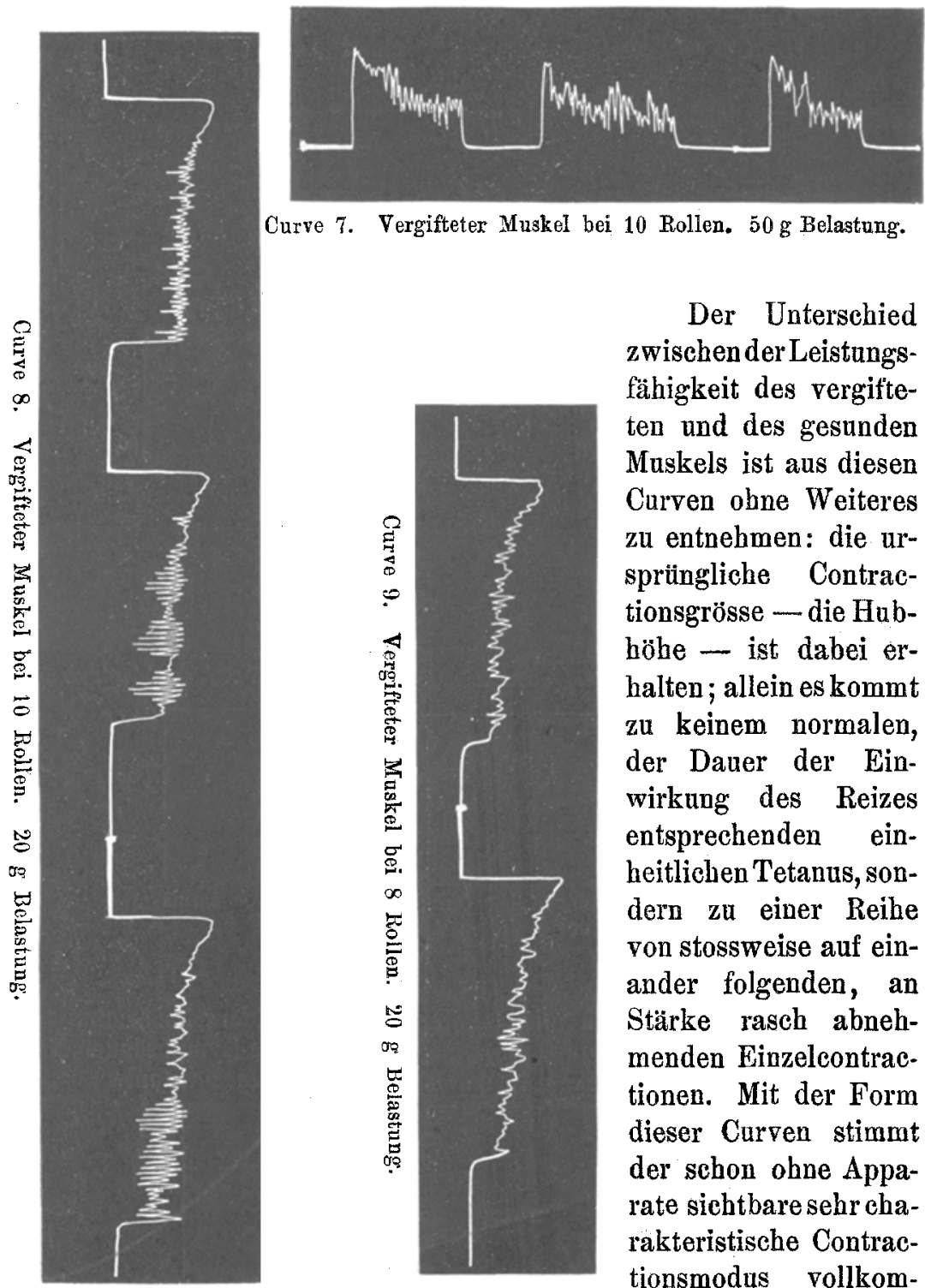

Curve 7. Vergifteter Muskel bei 10 Rollen. $50 \mathrm{~g}$ Belastung.

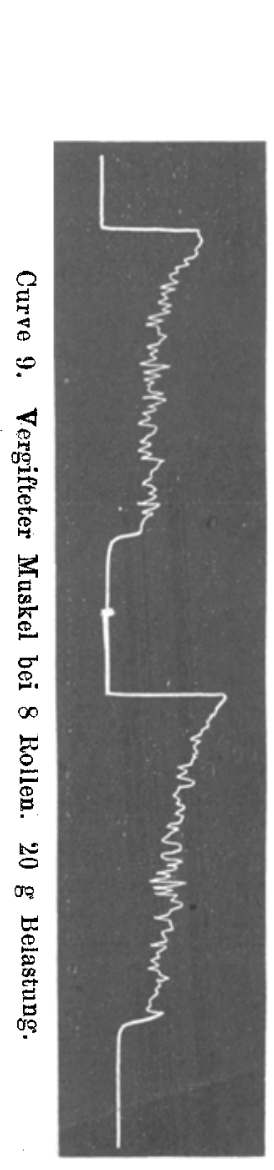

Der Unterschied 2wischen der Leistungsfähigkeit des vergifteten und des gesunden Muskels ist aus diesen Curven obne Weiteres zu entnehmen: die urspriingliche Contractionsgrösse - die Hubhöhe - ist dabei erhalten; allein es kommt zu keinem normalen, der Dauer der Einwirkung des Reizes entsprechenden einheitlichen Tetanus, sondern zu einer Reihe von stossweise auf einander folgenden, an Stärke rasch abnehmenden Einzelcontractionen. Mit der Form dieser Curven stimmt der schon obne Apparate sichtbare sehr eharakteristische Contractionsmodus vollkommen uiberein: immer bemerkt man eigenthümlich springende oder zitternde, wellen- oder wurmförmig über einzelne Bündel der grossen Muskeln sich erstreckende Einzelcontractionen.

Combinirt ist diese lähmende Wirkung auf die Muskelsubstanz mit einer gleichen auf die nervösen Endapparate. Immer ist gleich- 
zeitig die Erregbaikeit vom Nerven aus stark herabgesetzt, ja sie sinkt bis zum Erlöschen, wie der folgende Versuch zeigt.

\section{Versueh VII.}

Temporaria. Rechtes Bein mit Schonung des Ischiadicus unterbunden.

$6 \mathrm{~h}$. $2 \mathrm{~m}$. Injection von $4 \mathrm{mg}$ Chelidoniumprotopin.

$6 \mathrm{~h} .20 \mathrm{~m}$. Reiz mit dem Inductionsstrom bei 10 Rollen Distanz vom Nerven aus: Rechts Tetanus, links schwache zuckende Bewegungen.

6 h. $40 \mathrm{~m}$. Erregbarkeit links vom Nerven aus vollkommen erloschen, rechts erhalten.

Das Verbalten des Protopins zur Reflexfunction scheint auf den ersten Blick nicht ohne Widersprüche zu sein. Während nämlich die Reflexerregbarkeit bei kleinen und mittleren Dosen im Allgemeinen unbeeinflusst bleibt, bei grösseren aber entschieden rapid sinkt, findet sich hier und da bei mittelgrossen Gaben ein Zustand, der an eine gesteigerte Reflexerregbarkeit erinnert. Allein es kommt dabei niemals, man mag die Gaben in den weitesten Grenzen - von 2 mg bis $3 \mathrm{cg}$ - abstufen, zu wirklichen tetanischen Erscheinungen, sondern nur zu den bereits oben geschilderten, wobei einzelne Streckbewegungen mit vollständiger Erschöpfung der Reflexerregbarkeit abwechseln. Hat man die Gabe grösser gewählt, etwa $6 \mathrm{mg}$, so tritt schon nach ca. 20 Minuten complete Bewegungslosigkeit, Erloschensein aller Reflexe mit Stillstand der Athmung ein. Es besteht daher in diesem Punkte eine Analogie zwischen dem Protopin und den durch Säureeinwirkung entstandenen Thebainderivaten, dem Thebenin und Thebaicin, bei welch ersterem die Reflexerregbarkeit von vornherein bis zur Lähmung sinkt, während bei dem letzteren der Unerregbarkeit nicht selten ein Zustand vorhergeht, wo ,auf Reizung einige innormal heftige Extensionsbewegungen, welche reflectorischen Streckkrämpfen einigermaassen ähnlich sind“, erfolgen. ${ }^{1)}$ Zur weiteren Prüfung dieses Punktes wurde eine Anzahl mit Strychnin combinirter Vergiftungen vorgenommen.

\section{Versuch VIII.}

Esculenta. Seit 3 Tagen in Strychnintetanus.

11 h. 25 m. Auf den geringsten Reiz, z. B. Klopfen auf die Glocke, heftiger Reflextetanus.

$11 \mathrm{~h} .28 \mathrm{~m}$. Unterbindung der Arteria iliaca dextra. Injection von $6 \mathrm{mg}$ Opiumprotopin.

$11 \mathrm{~h} .35 \mathrm{~m}$. Reflexerregbarkeit noch unverändert.

$11 \mathrm{~h} .42 \mathrm{~m}$. Beiderseits noch typischer Tetanus.

1) W. v. Schröder, Untersuchnngen über die pharmakologische Gruppe des Morphins. Dieses Archiv. XVII. Bd. S. 139. 
11 h. $53 \mathrm{~m}$. Bereits geringer Unterschied zwischen beiden Beinen; rechts etwas intensivere Zuckung.

$11 \mathrm{~h} .55 \mathrm{~m}$. Die Reflexerregbarkeit nimmt rasch ab; es erfolgt auf Reizung in dem abgebundenen Beine zwar kein Tetanus, aber kräftige einmalige Zuckung, während in dem anderen nur rudimentäre Zuckung: eintritt.

$12 \mathrm{~h} .-$ m. Reflexerregbarkeit fast erloschen; nur in dem abgebundenen Bein ganz schwache Zuckung.

Auch aus diesen Versuchen geht mit Bestimmtheit hervor, dass bei grossen Dosen die Reflexthätigkeit aufgehoben ist.

Zur Charakterisirung der Wirkungsweise des Protopins auf das Froschherz möge ein Versuch von vielen gleichsinnigen folgen.

Versuch IX.

Temporaria-Männchen, in Rückenlage gefesselt, Herz blossgelegt.

$11 \mathrm{~h} .14 \mathrm{~m} .58$ Contractionen in der Minute.

$11 \mathrm{~h} .17 \mathrm{~m}$. Injection von $6 \mathrm{mg}$ Chelidoniumprotopin in die Seitenlymphsäcke. .

$11 \mathrm{~h} .21 \mathrm{~m} .46$ Contractionen in der Minute.

$11 \mathrm{~h} .24 \mathrm{~m} .20$ langsam und mühsam verlaufende, aber vollkommene Systolen, längere diastolische Pausen.

11 h. $27 \mathrm{~m} .16$ Contractionen.

11 h. 30 m. 14 Contractionen.

11 h. $34 \mathrm{~m} .26$ unvollkommene und kleine Contractionen.

11 h. 40 m. 29 Contractionen.

$11 \mathrm{~h} .45 \mathrm{~m}$. Injection von $2 \mathrm{mg}$.

11 h. 46 m. 20 sehr unvollkommene Contractionen, das Herz ist ganz blass und klein geworden.

12 h. - m. 18 Contractionen von leicht wellenförmigem Typus.

12 h. 15 m. 14 Contractionen. Sinusreizung wirkungslos.

$12 \mathrm{~h} .37 \mathrm{~m} .14$ Contractionen. 1 Tropfen Atropin in die Nähe des Herzens gebracht.

12 h. $40 \mathrm{~m} .14$ Contractionen. tractionen.

$12 \mathrm{~h} .51 \mathrm{~m}$. 16 sehr ausgesprochen wellenförmig verlaufende Con-

$3 \mathrm{~h} .5 \mathrm{~m} .14$ schwache Contractionen.

3 h. 50 m. 18 schwache Contractionen.

Die Wirkungsweise des Protopins ist daher auf die Schlagfolge und auf die Art der Contraction gerichtet. Seine erste Wirkung ruft eine Verlangsamung der Aetion herror, die Frequenz sinkt von 58 auf 14 ; dabei sind jedoch die Contractionen vollkommen, die Systolen etwas langsam und mühsam verlaufend, die Diastolen veriängert. Nach $1 / 4-1 / 2$ Stunde steigt die Frequenz wieder auf 26-28; doch ist nun die Action, des Herzens eine veränderte: die systolen werden sehr unvollkommen, das Herz sehr klein und 
blass, und bald darauf nehmen seine Contractionen einen eigenthümlichen Charakter an, indem sie wellen- oder wurmförmig über den Herzmuskel verlaufen, sich häufig nur über einzelne Theile desselben erstreckend. In diesem Zustande kann das Herz viele Stunden weiter schlagen. Schon der ganze Charakter dieser höchst unregelmässigen Contractionen lässt darauf schliessen, dass es sich in der Hauptsache um eine Wirkung auf den Herzmuskel handelt. Unterstiitzt wird diese Auffassung durch die Wirkungslosigkeit von Atropin. Sebr auffallend ist die hochgradige Anämie des Herzens. Ob diese der Grund des Ausbleibens der Vaguswirkung ist, oder ob es sich um directe Lähmung der Hemmungsapparate im Herzen handelt, lässt sich nicht entscheiden.

\section{Versuche an Säugethieren.}

Versuch X. Meerschweinchen.

9 h. $57 \mathrm{~m}$. Subcutane Injection von $5 \mathrm{mg}$ Protopin (Chelidonium).

10 h. - m. Das Thier ist sehr unruhig, schiesst im Käfige herum, versucht an den Wänden emporzuklettern.

$10 \mathrm{~h} .12 \mathrm{~m}$. Läuft noch immer unruhig herum, quiekt heftig und macht von Zeit zu Zeit heftige schmatzende Bewegungen.

$10 \mathrm{~h} .15 \mathrm{~m}$. Die Unıuhe lässt nach.

10 h. $25 \mathrm{~m}$. Wieder vớllig normal.

\section{Versuch XI. Meerschweinchen.}

$3 \mathrm{~h} .11 \mathrm{~m}$. Subcutane Injection von $6 \mathrm{cg}$. Opiumprotopin. Fast unmittelbar nach der Injection bemächtigt sich des Thieres grosse Unruhe; es rennt heftig mit der Schnauze gegen die Käfigstangen; dabei ununterbrochene Kaubewegungen.

$3 \mathrm{~h} .14 \mathrm{~m}$. Eintritt eines ersten Krampfanfalles, ats Trismus und Nackenkrampf bestehend.

3 h. 18 m. Nenerlicher Anfall mit clonischen Zuckungen in den Vorderbeinen, Lauf bewegungen gleichend.

$3 \mathrm{~h} .20 \mathrm{~m}$. bis 4 h. $8 \mathrm{~m}$. In dieser Zeit hat das Thier 22 Anfälle von verschiedener Intensität, die jedoch alle mit den geschilderten Symptomen, insbesondere den Laufbewegungen beginnen, worauf bei den heftigen Anfällen gewöhnlich Ueberschlagen nach rückwärts und wildes Umhergeschleudertwerden folgt.

$4 \mathrm{~h} .8 \mathrm{~m}$. bis $4 \mathrm{~h} .33 \mathrm{~m}$. Krampffreie Pause; anfangs liegt das Thier unter den Zeichen höchster Erschöpfnng auf der Seite, dann versucht es zu kriechen, wobei es seitlich umfällt; bald jedoch schwinden die Erscheinungen, das Thier ist nur äusserst matt.

$4 \mathrm{~h} .33 \mathrm{~m}$. Neuerlicher Anfall, dem bis $5 \mathrm{~h} .20 \mathrm{~m}$. 6 weitere folgen. Beobachtung unterbrochen.

8 h. - m. Das Thier sitzt ruhig im Käfig. Am nächsten Morgen wird es todt gefunden. 
Aus diesen zwei aus einer grossen Anzahl von Beobachtungen herausgehobenen Beispielen geht hervor, dass die Wirkung des Protopins auf Säuger von der auf Kaltbluter sehr verschieden ist. Ein narkotisches Stadium ist niemals, auch nur andeutungsweise vorhanden; vielmehr treten fast unmittelbar nach der Einverleibung des Giftes Zeichen von Unruhe und Angst, also Erregungszustände in der psychischen Sphäre auf, obne dass jedoch jemals eine Andeutung vorhanden wäre, welche diese Erscheinungen mit einer gesteigerten Reflexerregbarkeit in Zusammenhang brächte. Anschreien, Schlagen auf den Käfig u. s. w. haben weder jetzt einen Einfluss, noch im Krampfstadium. Den bei stärkeren Gaben - für Meerschweinchen $4 \mathrm{cg}$, für Katzen $8 \mathrm{cg}$ bis $1 \mathrm{dg}$ - eintretenden stürmischen Krämpfen fehlt der Charakter von Reflexkrämpfen ebenfalls gänzlich; sie treten vollkommen unabhängig in sehr kurzen und ziemlich gleichmässigen Pausen - bei einer kräftigen Katze alle 10 Minuten - also periodisch ein; sind ganz besonders durch Lauf- und Sprungbewegungen in den vorderen Extremitäten charakterisirt, welche stets den Anfall einleiten und bei mässigen Graden mit Trismus und Nackenkrampf das ganze Bild ausmachen, während die böheren Grade mit ibrer bis zu 10 Minuten betragenden Länge und dem völlig ungeordneten, wilden Umhergeschleudertwerden auf dem Boden und bis zur Decke des Käfigs einen eminent epileptiformen Charakter tragen. Das ganze Vergiftungsbild zeigt also eine frappante Analogie zu den Erscheinungen der Camphervergiftung. ${ }^{1}$ )

Der Tod kann bei grossen Gaben - bei einem Meerschweinchen nach $1 \mathrm{dg}$ - im Anfalle durch Erstickung eintreten, oder die Vergiftung zeigt einen protrahirteren Verlauf, es folgen sich im Laufe von 2-10 Stunden eine überaus grosse Anzahl von Anfällen, welche die Kräfte des Thieres consumiren: während es anfangs in den krampffreien Pausen noch zusammengekauert sitzt, liegt es später völlig kraftlos in passiver Seitenlage, und schliesslich tritt der Tod durch Erschöpfung ein.

Die Verhältnisse der Circulation wurden vermittelst des Kymographion am curarisirten und am nicht curarisirten Thiere eruirt. Als Beispiel der ersteren Art diene

Versuch XII. Katze von $2500 \mathrm{~g}$. Tracheotomie, kinstliche Respiration. $4 \mathrm{mg}$ Curarin (Boe h $)$ in die rechte Vena saphena injicirt. Vagi beiderseits abgeschnürt und durchtrennt. Linke Carotis am Kymographion.

1) Schmiedeberg, Grundriss der Arzneimittellehre. S. 121-122. 


\begin{tabular}{|c|c|c|c|}
\hline Zeit & $\begin{array}{l}\text { Mittlerer } \\
\text { Druck }\end{array}$ & $\begin{array}{c}\text { Frequenz } \\
\text { in } \\
10 \mathrm{Sec} .\end{array}$ & Bemerkungen \\
\hline $11 \mathrm{~h} 52 \mathrm{~m}$ & 193 & 38 & \\
\hline $12 \mathrm{~h} 1 \mathrm{~m}$ & 156 & 17 & Vagusreizung rechts. \\
\hline $12 \mathrm{~h} 2 \mathrm{~m}$ & 188 & 23 & Vagusreizung links. \\
\hline $12 \mathrm{~h} 10 \mathrm{~m}$ & 175 & 38 & $\begin{array}{l}\text { Injection von } 2 \mathrm{eg} \text { Chelidoniumprotopin in die } \nabla \text {. } \\
\text { saphena. }\end{array}$ \\
\hline $12 \mathrm{~h} 10 \mathrm{~m}$ & 104 & 34 & \\
\hline $12 \mathrm{~h} 15 \mathrm{~m}$ & 234 & 35 & \\
\hline $12 \mathrm{~h} 17 \mathrm{~m}$ & 214 & 36 & \\
\hline $12 \mathrm{~h} 20 \mathrm{~m}$ & 204 & 34 & \\
\hline $12 \mathrm{~h} 21 \mathrm{~m}$ & 159 & 38 & Injection von $1 \mathrm{cg}$ Protopin. \\
\hline $12 \mathrm{~h} 21 \mathrm{~m}$ & 189 & 35 & \\
\hline $12 \mathrm{~h} 21 \mathrm{~m}$ & 176 & 34 & \\
\hline $12 \mathrm{~h} 24 \mathrm{~m}$ & 60 & 23 & Injection von $2 \mathrm{cg}$ Protopin. \\
\hline $12 \mathrm{~h} 25 \mathrm{~m}$ & 72 & 34 & \\
\hline $12 \mathrm{~h} 28 \mathrm{~m}$ & 68 & 29 & Aussetzen der künstlichen Respiration. \\
\hline $12 \mathrm{~h} 29 \mathrm{~m}$ & 179 & 33 & Wiedereinführung der kutustlichen Respiration. \\
\hline $12 \mathrm{~h} 34 \mathrm{~m}$ & 174 & 35 & \\
\hline $12 \mathrm{~h} 39 \mathrm{~m}$ & 217 & 43 & $\begin{array}{l}\text { Reizung des Rüekenmarks durch } 2 \text { in der Höhe der } \\
\text { letzten Halswirbel eingestochene Elektroden. }\end{array}$ \\
\hline $12 \mathrm{~h} 53 \mathrm{~m}$ & 161 & 35 & \\
\hline $1 \mathrm{~h} 14 \mathrm{~m}$ & 141 & 30 & \\
\hline $1 \mathrm{~h} 14 \mathrm{~m}$ & 125 & 27 & Aussetzen der künstlichen Respiration. \\
\hline $1 \mathrm{~h} 16 \mathrm{~m}$ & 136 & 31 & Vaguasreizung. \\
\hline $1 \mathrm{~h} 20 \mathrm{~m}$ & - & - & Schreiben auf der Abscissenaxe. \\
\hline
\end{tabular}

\section{Als Beispiel der Wirkungsweise am nicht curarisirten Thiere diene} Versuch XIII. Starke Katze von 2920 g. Tracheotomie; keine künstliche Athmung. Vagi durchtrennt.

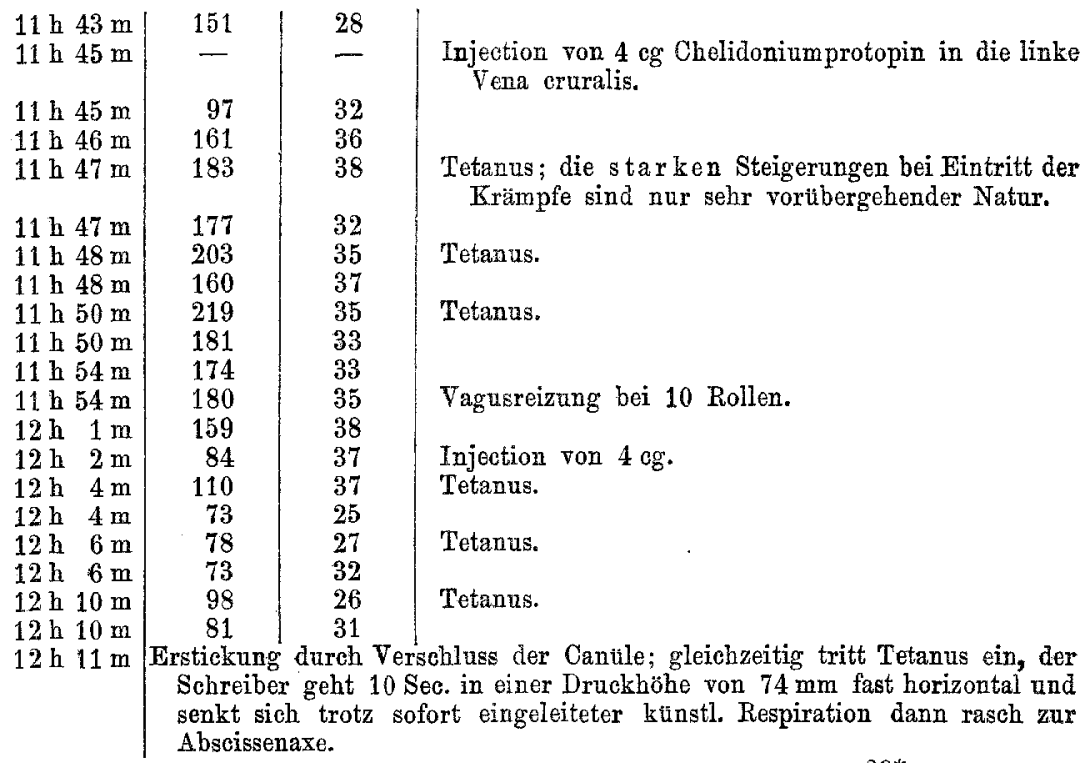


Schliesslich ein Beispiel der Wirkung grosser Gaben.

Versuch XIV. Kaninchen von 1720 g. Tracheotomie, beide Vagi armirt; in das Halsmark 2 Elektroden eingeführt.

\begin{tabular}{c|r|l}
\hline \hline \multicolumn{1}{c|}{ Zeit } & $\begin{array}{c}\text { Mittlerer } \\
\text { Druck }\end{array}$ & Bemerkangen \\
\hline $11 \mathrm{~h} 26 \mathrm{~m}$ & 102 & \\
$11 \mathrm{~h} 26 \mathrm{~m}$ & 99 & Subeutane Injection von $6 \mathrm{cg}$ Opiumprotopin. \\
$11 \mathrm{~h} 36 \mathrm{~m}$ & 106 & \\
$11 \mathrm{~h} 36 \mathrm{~m}$ & 104 & Subeutane Injection von $2 \mathrm{cg}$. \\
$11 \mathrm{~h} 43 \mathrm{~m}$ & 92 & \\
$11 \mathrm{~h} 44 \mathrm{~m}$ & 110 & Rückenmarksreiz bei 5 Rollen. \\
$11 \mathrm{~h} 51 \mathrm{~m}$ & 60 & Rüekenmarksreiz bei 4 Rollen. \\
$11 \mathrm{~h} 54 \mathrm{~m}$ & 106 & \\
$11 \mathrm{~h} 56 \mathrm{~m}$ & 70 & Verschluss der Trachealcanüle. \\
$11 \mathrm{~h} 57 \mathrm{~m}$ & 47 & Oeffnung der Trachealcanüle. Injection von 2 cg in die Vena \\
$11 \mathrm{~h} 58 \mathrm{~m}$ & 44 & jugularis externa sinistra. Der Druck sinkt binnen 18 Sec. \\
& & auf 12 und darauf unter unregeImüssigen Schwankungen zur \\
& & Abscisse.
\end{tabular}

Die in 2 Fällen sofort nach dem Stillstande der Circulation vorgenommene Se ctio n ergab folgenden Befund: Linkes Herz straff contrahirt, rechtes Herz schlaff. Die Herzohren zeigen fibrilläre Zuckungen. Vorhöfe auf tactile und elektrische Reize prompt erregbar, die Ventrikel in dem einen Falle vollkommen unerregbar, in dem anderen sind nur durch starke Ströme ganz leichte Vibrationen einzelner Bündel auszulösen.

Die Wirkung des Protopins auf die Circulation besteht demnach darin, dass bei kleinen und mittleren Dosen von 2, höchstens $4 \mathrm{cg}$ auf ein ganz voribergehendes Absinken des Druckes unmittelbar nach der Injection der Blutdruck steigt. Am nicht curarisirten Thiere treiben die einzelnen Tetani den Druck ganz voribergehend noch mehr in die Höhe. Die Wirkung ist jedoch eine transitorische, und der Druck geht bald zur Norm zurïck. Wiederholt man die Einverleibung des Giftes, so erhält man wieder momentanen Abfall und darauffolgendes Ansteigen; doch erreicht der Druck nicht mehr die alte Höhe, sinkt vielmehr immer tiefer; ebenso ist bei grösseren Dosen von 6-8 $\mathrm{cg}$ das Ansteigen nur ein ganz vorübergehendes und weicht einem allmählichen Sinken des Druckes.

Die Aussetzung der künstlichen, sowie der Abschluss der natürlichen Respiration hat nicht nur kein Ansteigen, sondern vielmehr stets ein ganz entschiedenes Absinken des Druckes im Gefolge. Vagusreizung blieb gänzlich ohne Effect. Hingegen hatte Reizung des Halsmarkes constant seine Wirksamkeit bewahrt. Sensible Reize wiederum blieben ohne Einfluss. 
Es ist daher die Wirknngsweise des Protopins einerseits auf das Herz, andererseits auf das Gefässnervencentrum gerichtet; in beiden Fällen lähmend, und zwar im Herzen schon bei mässigen Gaben die Hemmungsapparate, bei grösseren den Herzmuskel. Die peripheren Gefässnerven hingegen haben ihre Erregbarkeit bewahrt, man kann durch directe Reizung ihrer Bahnen im Rückenmark Drucksteigerung erzielen.

Ein besonderer Einfluss anf die Respiration ist nicht nachzuweisen. Im Anfang, zur Zeit der psychischen Erregung der Thiere, ist die Respirationsfrequenz natürlich gesteigert. An einem gefesselten Meerschweinchen, das $2 \mathrm{cg}$ subcutan erhalten hatte, wurde die Frequenz während und nach den Anfällen gezählt; sie bewegte sich zwischen 120-150 in 1 Minute. In dem Erschöpfungsstadium nach häufigen Anfällen pflegte die Athemfrequenz der Meerschweinchen ca. 180 zu betragen. Ebensowenig konnte ein Einfluss auf den Digestionstract und die Secretionen wahrgenommen werden.

Sollten zum Sehlusse die Wirkungen des Protopins auf den Organismus der Kalt- und Warmblïter zusammengefasst werden, so ergäben sich folgende Punkte:

1. Auf den Frosch wirkt das Protopin in kleinen Dosen gleich den meisten Opiumalkaloiden narkotisch.

2. In starken Gaben wirkt es auf die Muskelsubstanz, sowie auf die peripheren Nervenendigungen lähmend.

3. Die Reflexerregbarkeit ist bei kleinen und mittleren Gaben wohlerhalten, bei grossen aufgehoben.

4. Auf das Sängethier hat das Protopin eine der Camphervergiftung ähnliche Wirkung; doch unterscheidet sie sich von derselben durch die Lähmung der Kreislaufsorgane.

Marburg i/H., im April 1890. 